\title{
BEITRÄGE ZUR WIRKUNG DER DAUER DER MELKPAUSE AUF DIE INTENSITÄT DER MILCHSEKRETION DES KUHEUTERS
}

\author{
ILMARI POIJÄRVI \\ Landwirtschaftliche Versuchsanstalt, Abteilung für Haustierhaltung, Tikkurila.
}

Eingegangen am 20.12.1953.

Allgemein anerkannt dürfte heute die Auffassung sein, dass die Dauer der Melkpausen besonders auf die Milcherzeugung der hochmelkenden Kühe einwirkt. In den Grenzen, die praktisch in Frage kommen können, dürfte eine kurze Pause günstig, eine lange aber ungünstig auf die Milchsekretion einwirken $(2,7,8)$. Dies beruhe darauf, dass diejenige Zunahme im innerem Druck des Euters, die auf dem Anfüllen der Drüsenbläschen und Milchkanälchen mit abgesonderter Milch beruht, die Sekretion neuer Milch schliesslich ganz verhindert (3, 4, 9). Je häufiger das Euter entleert würde, desto weniger könne dieser die Milchbildung hemmende Faktor wirksam sein. Diese Auffassung scheinen u.a. die zahlreichen Versuche, die zum Vergleich zwei -und dreimaligen Melkens ausgeführt worden sind, zu bestätigen (vergl. z.B. 1 und 5). Kürzlich hat der Verfasser (6) jedoch auch solche Versuchsergebnisse veröffentlicht, die wenigstens auf den ersten Blick mit dieser Auffassung nicht im Einklang stehen. Bei zweimaligem Melken haben mehrere Kühe durchschnittlich dieselbe Milchleistung gegeben, unabhängig davon, ob die Pause zwischen Abend- und Morgenmelken 12 St, 13 St, 14 St oder 15 St ausgemacht hat, vorausgesetzt dass das Verlängern oder Verkürzen der Melkpausen sehr allmählich, nur 5 -8 Min täglich, vor sich gegangen ist.

Es ist jedoch denkbar, diese Ergebnisse so zu erklären, dass bei der Anwendung von ungleichen Melkpausen die Intensität der Milchsekretion sich während der kürzeren Pause, als eine Folge der Druckverminderung, genau in demselben Masse erhöht, als sie sich während der längeren Pause, als eine Folge der Drucksteigerung, senkt.

Auf Grund des Versuchsmaterials, das in den oben erwähnten zwei Versuchen des Verfassers gesammelt worden ist, ist es möglich, auch die Intensität der Milchsekretion während der verschieden langen Melkpausen zu untersuchen. Die Melkpausen dauerten nämlich mindestens bei 6 Kühen und 2 Wochen genau 9, 10, 11,12 und entsprechend 15, 14, 13 und 12 Stunden. Nach dem Melkakt wurde die Milch- 
menge immer gewogen. Es ist also möglich, in allen Fällen die Milchleistung in einer Zeiteinheit zu berechnen.

Die gelegentlichen Schwankungen in den Tagesleistungen einer Kuh sind ja bekanntlich sehr gross. Um eine richtige Auffassung von der Intensität der Milchsekretion zu erhalten, ist es darum notwendig, die Leistungen während eines längeren Zeitabschnittes zu beachten. Das wird auch in den folgenden Betrachtungen geschehen. Die Perioden mit den konstanten Melkpausen haben wenigstens 15 Tage umfasst.

\section{Die Wirkung der Dauer der Melkpause auf die Intensität der Milchsekretion}

Allgemeine Anordnung der Versuche. Um die folgenden Berechnungen und Darlegungen verstehen zu können, ist es nötig, etwas von der Ausführung der Versuche zu wissen, bei denen das Grundmaterial gesammelt worden ist.

Unter Berücksichtigung der bei einem Vorversuch gesammelten Erfahrungen wurde bei den jetzt zu beschreibenden Versuchen der Übergang von einer Melkzeit zur anderen sehr langsam durchgeführt. Bei dem ersten Versuch, der mit reichlicher melkenden Kühen angestellt wurde, verlängerte (oder verkürzte) man die Melkpausen nur um 5 Min. täglich und bei dem zweiten Versuch um 8 Min. täglich.

Beide Versuche dauerten 90 Tage und verteilten sich auf drei gleich lange Perioden. Die Fütterung der Kühe, die nach ihrem Lebendgewicht und nach der zu Beginn des Versuchs festgestellten Leistung sich richtete, blieb bis zum Ausgang des Versuchs dieselbe. Nur die Beschaffenheit des AIV-Futters wechselte in gewissem Masse, besonders bei dem letzteren Versuch. Die im AIV-Futter gegebene Nährstoffmenge versuchte man jedoch unter Berücksichtigung der chemischen Zusammensetzung möglichst gleichwertig zu halten. Ausserdem konnten etwaige Wirkungen der Fütterung dadurch beurteil werden, dass beide Versuche zwei Gruppen umfassten und dass die eine Gruppe gleichzeitig zu einer kürzeren nächtlichen Melkpause überging, während in den Melkzeiten der anderen eine entgegengesetzt gerichtete Veränderung eintrat.

Bei beiden Versuchen ging man von so gelegenen Melkzeiten aus, dass jede Versuchskuh morgens genau 13 Stunden nach dem Abendmelken gemolken wurde, so dass für die Melkpause am Tage 11 Stunden blieben. Ein derartiges Verfahren ist heute in Finnland sehr allgemein.

Da sich in der Praxis der Arbeitstag der Tierpfleger schon recht angemessen gestalten liesse, wenn die Nachtpause der am reichlichsten melkenden Kühe ohne Nachteil z.B. 14 St und bei mittelmässigem Melken $15 \mathrm{St}$ ausmachen könnte, wurde beschlossen, gerade diese Zeiten in Versuchen zu prüfen. Zugleich sollte aber auch die etwaige Wirkung einer möglichst kurzen oder 12stündigen Nachtpause auf die Milcherzeugung in die Versuche einbezogen werden.

Der erste Versuch wurde mit 12 Kühen, deren durchschnittliche Tagesleistung anfangs $21.5 \mathrm{~kg}$ betrug, ausgeführt. Sie wurden in zwei Gruppen von je 6 Kühen geteilt. Der allgemeine Plan des Versuches geht aus dem folgenden Schema hervor: 


$\begin{array}{cccc} & \begin{array}{c}\text { 1. Periode }(30 \mathrm{Tg}) \\ \text { Nachtpause }\end{array} & \begin{array}{c}\text { 2. Periode }(30 \mathrm{Tg}) \\ \text { Nachtpause }\end{array} & \begin{array}{c}\text { 3. Periode }(30 \mathrm{Tg}) \\ \text { Nachtpause }\end{array} \\ \begin{array}{c}\text { Gruppe I } \\ \text { II }\end{array} & 13 \mathrm{St} & (13 \mathrm{St})-14 \mathrm{St} & (14 \mathrm{St})-13 \mathrm{St} \\ 13 \mathrm{St} & (13 \mathrm{St})-12 \mathrm{St} & (12 \mathrm{St})-13 \mathrm{St}\end{array}$

Die Pause vom Abend- bis zum Morgenmelken wurde bei diesem Versuch bei Gruppe I so verlängert, dass anfangs das Abendmelken täglich um 5 Min. früher verlegt wurde, bis es um $1 / 2$ St verschoben war. Danach wurde morgens täglich 5 Min. später gemolken, bis die Nachtpause 14 St dauerte. Dies wurde also in 12 Tagen erreicht. - Bei Gruppe II wurde die Nachtpause durch entsprechendes Vorgehen auf 12 St verkürzt.

Der zweite Versuch wurde unmittelbar nach dem vorhergehenden grösstenteils mit denselben Tieren ausgeführt. Die mittlere Milchleistung der Gruppen belief sich anfangs auf 16.6 und $16.7 \mathrm{~kg}$. Der allgemeine Versuchsplan geht aus dem folgenden Schema hervor:

\begin{tabular}{|c|c|c|c|c|}
\hline & & $\begin{array}{l}\text { 1. Periode }(30 \mathrm{Tg}) \\
\text { Nachtpause }\end{array}$ & $\begin{array}{l}\text { 2. Periode }(30 \mathrm{Tg}) \\
\text { Nachtpause }\end{array}$ & $\begin{array}{l}\text { 3. Periode }(30 \mathrm{Tg}) \\
\text { Nachtpause }\end{array}$ \\
\hline Gruppe & I & $13 \mathrm{St}$ & $(13 \mathrm{St})-15 \mathrm{St}$ & $(15 \mathrm{St})-13 \mathrm{St}$ \\
\hline , & II & $13 \mathrm{St}$ & $(13 \mathrm{St})-12 \mathrm{St}$ & $(12 \mathrm{St})-13 \mathrm{St}$ \\
\hline
\end{tabular}

Die Verlängerung der Nachtpause wurde bei Gruppe I so durchgeführt, dass anfangs das Abendmelken täglich 8 Min. früher verlegt wurde, bis die Pause in der Nacht etwa 14 St ausmachte. Danach begann man das Morgenmelken täglich 8 Min. später vorzunehmen, bis eine 15stündige Nachtpause erreicht wurde. Für den Übergang waren also insgesamt 15 Tage erforderlich. - Bei Gruppe II wurde die Nachtpause auf dieselbe Weise wie beim vorhergehenden Versuch auf 12 Stunden verkürzt.

In den folgenden Berechnungen sind die Übergangstage selbstverständlich nicht in Betracht gezogen worden.

Die Verteilung der Milchleistung auf Morgen- und Abendmelken bei wechselnder Dawer der Melkpausen. Wäre die Milchsekretion im Euter ein kontinuierlicher Vorgang, also z.B. die im Verlaufe jeder Minute abgesonderte Milchmenge 24 Stunden lang dieselbe, so erhielte man natürlich bei 12stündiger Melkpause beim Morgenund beim Abendmelken eine gleich grosse Milchmenge. Machte die Melkpause in der Nacht 13 Stunden aus und die am Tage 11 St, so erhielte man beim Abendmelken unter gleicher Voraussetzung 11/24 oder $45.83 \%$ der gesamten Tagesleistung, bei 10stündiger Tagespause 10/24 oder $41.67 \%$, bei 9 stündiger $9 / 24$ oder $37.50 \%$ usw. Wenn wiederum, nach den Theorien, die Verlängerung der Melkpause infolge der auf dem stärkeren Milchdruck beruhenden Hemmung zu einer Verminderung der Milchabsonderung in der Zeiteinheit führte, so erhielte man bei über 12stündiger Pause in der Nacht und bei kürzerer am Tage beim Abendmelken einen verhältnismässig grösseren Anteil an der gesamten Tagesleistung, als oben berechnet worden ist. Je länger die Pause in der Nacht und je kürzer die am Tage wäre, desto mehr müsste z.B. der prozentuale Anteil der beim Abendmelken erhaltenen Milchmenge von den obigen Werten abweichen. 


\begin{tabular}{|c|c|c|c|c|c|c|c|c|}
\hline \multirow{2}{*}{\multicolumn{2}{|c|}{$\begin{array}{c}\text { Dauer } \\
\text { der } \\
\text { Nacht- } \\
\text { pause } \\
\text { St }\end{array}$}} & \multicolumn{3}{|c|}{ Gesamtmilchleistung } & \multicolumn{3}{|c|}{$\begin{array}{c}\text { Abendleistung \% der } \\
\text { Gesamtleistung }\end{array}$} & \multirow{2}{*}{$\begin{array}{l}\text { Mittl. } \\
\text { Tages- } \\
\text { leistung } \\
\text { kg }\end{array}$} \\
\hline & & $\begin{array}{l}\text { bei allem } \\
\text { Abend- } \\
\text { melken } \\
\text { kg }\end{array}$ & $\begin{array}{l}\text { bei allem } \\
\text { Morgen- } \\
\text { melken } \\
\text { kg }\end{array}$ & $\begin{array}{l}\text { insgesamt } \\
\text { kg }\end{array}$ & $\begin{array}{l}\text { theoretisch } \\
\text { berechne- } \\
\text { ter Wert }\end{array}$ & $\begin{array}{l}\text { wirklich } \\
\text { erhalten }\end{array}$ & $\begin{array}{l}\text { Extrem- } \\
\text { werte }\end{array}$ & \\
\hline \multicolumn{9}{|c|}{ Gruppe I } \\
\hline 1. Periode & 13 & 1178.9 & 1374.2 & 2553.1 & 45.83 & 46.18 & $45.44-47.96$ & 21.27 \\
\hline $2 . \quad$ & 14 & 973.2 & 1376.0 & 2349.2 & 41.67 & 41.43 & $39.50-43.11$ & 19.58 \\
\hline 3. & 13 & 1000.7 & 1195.8 & 2196.5 & 45.83 & 45.56 & $44.93-46.33$ & 18.30 \\
\hline \multicolumn{9}{|c|}{ Gruppe II } \\
\hline 1. Periode & 13 & 1194.4 & 1401.5 & 2595.9 & 45.83 & 46.01 & $45.11-47.06$ & 21.63 \\
\hline $2 . \quad$ & 12 & 1207.7 & 1216.1 & 2423.8 & 50.00 & 49.83 & $48.89-50.98$ & 20.20 \\
\hline 3. & 13 & 1044.1 & 1245.1 & 2289.2 & 45.83 & 45.61 & $45.07-46.82$ & 19.08 \\
\hline
\end{tabular}

Von diesen einfachen Tatsachen ausgehend, wird im folgenden die Frage betrachtet, ob die Verlängerung oder Verkürzung der Melkpause in den Grenzen, wie sie in den Versuchen in Frage gekommen sind, Veränderungen in der Intensität der Milchabsonderung bewirkt hat, ob, mit anderen Worten, die in der Zeiteinheit gebildete Milchmenge sich bei wechselnder Melkzpause verändert hat.

Zur Klärung der Frage ist für beide Versuche und gesondert für jede ihrer Perioden berechnet worden, wieviel Milch man insgesamt bei allem Morgenmelken und wieviel bei allem Abendmelken erhalten hat. Danach ist berechnet worden, wieviel Prozent die bei allem Abendmelken erhaltene Milchmenge von der Gesamtmilchmenge ausmacht. Der erhaltene Prozentsatz ist verglichen worden mit dem, der sich herausstellt, wenn die Milchabsonderung als ganz gleichmässig während 24 Stunden vorausgesetzt wird. Die Ergebnisse sind aus den Tabellen 1 (erster Versuch) und 2 (zweiter Versuch) zu ersehen.

Bei Tabelle 1 haben den Berechnungen die Milchleistungen der letzten 20 Tage jeder Versuchsperiode zugrunde gelegen. Bei Tabelle 2 wiederum gründen sich die Berechnungen auf die Leistungen der letzten 15 Tage.

Wird auf Grund der in den Tabellen 1 und 2 angegebenen Mittelwerte der ganzen Gruppen die Wirkung des Wechsels der Melkpause auf die Intensität der Milchabsonderung beurteilt, so kommt man zu nachstehenden Schlussfolgerungen.

Wenn die Pause in der Nacht und die am Tage gleich lang gewesen sind, also 12 St, hat man beide Male beim Melken so gut wie $50 \%$ der gesamten Tagesleistung erhalten. Bei dem Versuch mit reichlicher melkenden Kühen erhielt man beim Abendmelken $49.8 \%$ und bei dem mit weniger melkenden Tieren wiederum $50.2 \%$.

Die 13stündige Pause in der Nacht hat auch in keinem einzigen Falle die Milchabsonderung deutlich gehemmt, da die beim Abendmelken erhaltenen Milchmengen in allen Fällen den theoretischen Werten, die eine gleichmässige Sekretion voraussetzen, erstaunlich gleichgekommen sind. Berechnet man den Mittelwert für alle 
Tabelle 2 .

\begin{tabular}{|c|c|c|c|c|c|c|c|c|}
\hline & \multirow{2}{*}{$\begin{array}{l}\text { Dauer } \\
\text { der } \\
\text { Nacht- } \\
\text { pause } \\
\text { St }\end{array}$} & \multicolumn{3}{|c|}{ Gesamtmilchleistung } & \multicolumn{3}{|c|}{$\begin{array}{c}\text { Abendleistung \% der } \\
\text { Gesamtleistung }\end{array}$} & \multirow{2}{*}{$\begin{array}{l}\text { Mittl. } \\
\text { Tages- } \\
\text { leistung } \\
\text { kg }\end{array}$} \\
\hline & & $\begin{array}{l}\text { bei allem } \\
\text { Abend- } \\
\text { melken } \\
\text { kg }\end{array}$ & $\begin{array}{c}\text { bei allem } \\
\text { Morgen- } \\
\text { melken } \\
\text { kg }\end{array}$ & $\begin{array}{c}\text { insgesamt } \\
\text { kg }\end{array}$ & $\begin{array}{l}\text { theoretisch } \\
\text { berechne- } \\
\text { ter Wert }\end{array}$ & $\begin{array}{l}\text { wirklich } \\
\text { erhalten }\end{array}$ & $\begin{array}{l}\text { Extrem- } \\
\text { werte }\end{array}$ & \\
\hline \multicolumn{9}{|l|}{ Gruppe I } \\
\hline 1. Periode & le 13 & 677.6 & 800.6 & 1478.2 & 45.83 & 45.84 & $45.20-46.83$ & 16.42 \\
\hline $2 . \quad$ & 15 & 556.7 & 905.5 & 1462.2 & 37.50 & 38.07 & $36.62-40.95$ & 16.25 \\
\hline 3. & 13 & 615.0 & 728.6 & 1343.6 & 45.83 & 45.77 & $45.12-46.67$ & 14.93 \\
\hline \multicolumn{9}{|l|}{ Gruppe II } \\
\hline 1. Periode & le 13 & 658.4 & 782.8 & 1441.2 & 45.83 & 45.68 & $45.30-46.06$ & 16.01 \\
\hline $2 . \quad$, & 12 & 719.0 & 713.8 & 1432.8 & 50.00 & 50.18 & $49.24-52.40$ & 15.92 \\
\hline 3. & 13 & 621.4 & 733.2 & 1354.6 & 45.83 & 45.87 & $44.85-46.18$ & 15.05 \\
\hline
\end{tabular}

acht Fälle ganzeı Gruppen und Perioden, bei denen eine Nachtpause von 13 Stunden eingehalten worden ist, so ergibt sich, dass der Anteil der beim Abendmelken gewonnenen Milch $45.83 \%$ der gesamten täglichen Milchmenge ausmacht oder also genau denselben Wert, der durchaus gleichmässiger Sekretion entspricht.

Zur Beleuchtung des Sachverhalts seien ferner die Prozentsätze für das Abendmelken verschieden stark melkender Kühe bei Einhaltung 13stündiger Nachtpause berechnet. Derartige Fälle waren in der Mehrzahl oder 48. Werden sie nach der Menge der Leistung z.B. in sechs Gruppen eingeteilt, so ergibt sich folgende $\mathrm{Zu}-$ sammenstellung.

\begin{tabular}{|c|c|c|c|c|c|}
\hline & & \multicolumn{2}{|c|}{ Tagesleistung } & \multirow{2}{*}{$\begin{array}{c}\text { Das Abendmelken } \\
\% \text { von der } \\
\text { Gesamtleistung }\end{array}$} \\
\hline & & & $\begin{array}{c}\text { im Mittel } \\
\mathrm{kg}\end{array}$ & $\begin{array}{l}\text { Extremwerte } \\
\mathrm{kg}\end{array}$ & \\
\hline & Gruppe, 8 & 8 Fälle & 23.53 & $22.24-27.33$ & 45.65 \\
\hline 2. & " & " & 21.07 & $20.11-22.21$ & 45.98 \\
\hline 3. & $"$ & " & 18.71 & $17.70-20.00$ & 45.74 \\
\hline 4. & $"$ & " & 16.27 & $15.49-17.44$ & 45.88 \\
\hline 5. & " & " & 14.65 & $14.40-15.18$ & 45.94 \\
\hline 6. & $"$ & " & 12.85 & $11.96-14.03$ & 45.87 \\
\hline
\end{tabular}

Aus der Zusammenstellung geht anschaulich hervor, dass selbst die Milchsekretion der am reichlichsten melkenden Kühe nicht unter einer 13stündigen Nachtpause gelitten hat. Die kleinen Unterschiede, die zwischen den verschiedenen Gruppen bestehen, folgen durchaus nicht dem Leistungsbetrage, so dass sie auf reinem Zufall beruhen.

In dem ersten Versuch gab Gruppe I beim Melken durchschnittlich $19.58 \mathrm{~kg}$ täglich, bei Einhaltung von 14stündiger Nachtpause beim Abendmelken $41.47 \%$ der gesamten Milchmenge von 24 Stunden, während sich der einer gleichmässigen Sekretion entsprechende Wert auf $41.67 \%$ belief. Theoretisch hätte sie bei nur 
10stündiger Pause am Tage mehr Milch in der Zeiteinheit geben müssen als in 14stündiger Nachtpause. Das Verhältnis war aber umgekehrt, wenn auch der Unterschied so gering ist, dass er kaum als sicher gelten kann, wie sich später zeigen wird. Meistberechtigt erscheint die Schlussfolgerung, dass eine 14stündige Pause in der Nacht in der Milchabsonderung von Kühen mit einer täglichen Milchleistung von $19.6 \mathrm{~kg}$ noch keine Störungen verursacht, vielmehr setzt sich diese durchaus gleichmässig fort.

Die mittleren Leistungen der einzelnen Kühe und die entsprechenden Prozentsätze der Abendmilch bei Einhaltung einer 14stündigen Nachtpause waren nach dem Betrage angeordnet folgende:

\begin{tabular}{|c|c|c|}
\hline Name der Kuh & Milchleistung & Prozentsatz der Abendmilch \\
\hline Riisto ...... & $23.34 \mathrm{~kg}$ & 41.69 \\
\hline Hämärä ............ & 21.62 & 42.55 \\
\hline Esikki & 20.88 & 39.50 \\
\hline Nasti $\ldots .$. & 17.90 & 40.29 \\
\hline Alli $\ldots \ldots \ldots \ldots \ldots \ldots$ & 17.56 & 43.11 \\
\hline Hapsi . . . . . . . . . . . . & 16.17 & 41.47 \\
\hline
\end{tabular}

Die obige Zusammenstellung lässt erkennen, dass eine solche Regelmässigkeit, dass die Milchsekretion der am meisten melkenden Kühe unter 14stündiger Nachtpause mehr gelitten hätte als die der wenig melkenden, nicht wahrzunehmen ist. Die kleinen Schwankungen, die in den Prozentsätzen des Abendmelkens zu beobachten sind, beruhen auf blossem Zufall.

Die längste Pause in der Nacht bei diesen Versuchen betrug 15 Stunden, wobei ide Pause am Tage also nur 9 St ausmachte. In dem Versuch mit weniger melkenden Kühen gab Gruppe I bei durchschnittlicher täglicher Milchmenge von $16.25 \mathrm{~kg}$ nach 9stündiger Tageszeit eine Milchleistung, die $38.07 \%$ der gesamten Tagesleistung ausmachte, während sich der einer gleichmässigen Milchsekretion entsprechende Wert auf $37.50 \%$ belief. Der Unterschied ist auch in diesem Fall also recht gering, wenn auch grösser als in dem vorhergehenden und so gerichtet, dass er auf eine geringe Verlangsamung der Milchsekretion während einer langen Nachtzeit gegenüber der in kurzer Tageszeit vor sich gegangenen Milchabsonderung hinweisen könnte. Es wäre demnach theoretisch interessant, Klarheit darüber zu gewinnen, ob der betreffende Unterschied wirklich eine Folge der eben genannten Erscheinung oder nur zufallsbedingt ist.

Aufschluss über diese Frage gibt folgende Zusammenstellung, in der die Kühe, die mit Pausen von 15 und 9 Stunden gemolken wurden, nach der Reichlichkeit der Milchleistung aufgeführt sind.

\begin{tabular}{|c|c|c|}
\hline Name der Kuh & Milchleistung & Prozentsatz der Abendmilch \\
\hline Riisto $\ldots \ldots \ldots \ldots \ldots$. & $19.34 \mathrm{~kg}$ & 37.02 \\
\hline Uusikki $\ldots \ldots \ldots \ldots \ldots$ & 18.70 & 38.07 \\
\hline Ruuti $\ldots \ldots \ldots \ldots \ldots$. & 16.40 & 37.46 \\
\hline Alli $\ldots \ldots \ldots \ldots \ldots \ldots$. & 15.19 & 38.83 \\
\hline Nasti $\ldots \ldots \ldots \ldots \ldots$ & 14.18 & 36.62 \\
\hline Esikki .. & 13.66 & 40.95 \\
\hline
\end{tabular}


Aus der Zusammenstellung geht hervor, wie offenbar ein Zufall dazu geführt hat, dass für das Abendmelken, wie oben ersichtlich, eine etwas grössere Milchleistung als die einer gleichmässigen Milchsekretion entsprechende Menge erhalten worden ist. Wenn nämlich eine auf Enge beruhende Hemmung im Euter die Ursache dazu gewesen wäre, so wäre die Wirkung um so deutlicher hervorgetreten, je reichlicher die Kuh melkt. Das ist nicht der Fall gewesen, vielmehr ist zufällig der Prozentsatz des Abendmelkens der am reichlichsten melkenden Kuh der zweitkleinste gewesen, während wiederum das entsprechende Prozent der am wenigsten melkenden Kuh am grössten gewesen ist.

Auf Grund der im Versuch erhaltenen Leistungen kann also gefolgert werden, dass wenigstens bei $14-19 \mathrm{~kg}$ - melkenden, $450-550 \mathrm{~kg}$ wiegenden Kühen nicht einmal eine so lange wie 15stündige Melkpause zu einem solchen Milchdruck im Euter führen kann, dass er hemmend auf die in einer Zeiteinheit entstandene Milchmenge, also auf die Intensität der Milchsekretion einwirken könnte.

\section{Besprechung der Ergebnisse}

Die oben beschriebenen Versuche, die mit 450—550 kg schweren Ayrshirekühen guter Erzeugungsfähigkeit ausgeführt worden sind, haben zu dem Ergebnis geführt, dass die Intensität der Milchsekretion oder also die in der Zeiteinheit abgesonderte Milchmenge durch die verschieden langen Melkpausen nicht beeinflusst wurde.

Dass die bei diesen Versuchen erhaltenen, oben herausgestellten Ergebnisse in scheinbarem Widerstreit mit vielen früher gewonnenen Resultaten stehen, lässt sich offenbar dadurch erklären, dass bei diesen Versuchen die Veränderungen in den Melkpausen nicht so gross gewesen sind wie z.B. beim Übergang vom dreimaligem zum zweimaligen Melken und trotzdem der Übergang von einer Melkpause zur anderen stets sehr allmählich vor sich gegangen ist. In den meisten Fällen wurde die Melkpause nur um 5 Min. täglich verlängert und entsprechend verkürzt, in einem Falle um 8 Min./Tg. So liess sich zunächst eine Beunruhigung der Kühe vermeiden - derartige Schwankungen kommen ja in der Praxis ununterbrochen vor, so dass die Kühe daran gewöhnt sind - , und vor allem wurde dem Euter genügend Zeit gelassen, sich den neuen Verhältnissen anzupassen.

Damit der letztere Sachverhalt die in den Versuchen erhaltenen von den früheren abweichenden Ergebnisse erkläre, müsste das Euter grössere Möglichkeiten haben, sich dem "Speichern" verschieden reichlicher Milchmengen anzupassen," als bisher angenommen worden ist. Nach Verfassers Ansicht verhält es sich auch so.

Zunächst ist es offenbar, dass höchstens einmal im Leben jeder Kuh die gesamte Kapazität ihres Euters ausgenutzt wird, nämlich dann, wenn sie die reichlichste Einmalleistung ihres Lebens gibt. Gewiss ist es keineswegs sicher, dass selbst dann die maximale Kapazität des Euters ausgenutzt wird, aber gewiss ist, dass ihr Euter wenigstens soviel fertige Milch fassen kann (zuzüglich des Rohstoffes der während des Melkens vielleicht abgesonderten Milch). Wir können also dessen gewiss sein, dass die Kuh alle übrigen Male, soviel an der Euterkapazität liegt, mehr Milch hätte geben können, als sie gegeben hat. Dass die Leistung auf der Stufe haltmacht, 
auf der sie haltgemacht hat, beruht also auf anderen Ursachen als Raummangel. Die wichtigste von ihnen ist wahrscheinlich die Sekretionsmenge der die Milchabsonderung regelnden Hormone, dann folgen Menge und Beschaffenheit der für die Produktion zur Verfügung stehenden Nährstoffe, welche ihrerseits vielleicht auf die Hormonsekretion einwirken, ferner die Melkintensität usw.

Es kann also als wahrscheinlich gelten, dass es im Euter stets auch solche Abteilungen gibt, die nicht tätig sind, da sie nicht notwendig sind für die Absonderung und Speicherung derjenigen Milchmenge, deren Zustandekommen in erster Linie jeweils die Hormone bestimmen. Verändern sich die Verhältnisse in einer oder der anderen Weise dahin, dass mehr tätige Euterdrüse erforderlich ist, so ist es wenigstens theoretisch möglich und meiner Meinung nach natürlich, dass die erforderliche Menge vorrätigen Raumes benutzt wird. Das kann wohl aber nicht augenblicklich geschehen, sondern es mag eine kürzere oder längere Zeit in Anspruch nehmen, bis die in Ruhe befindlichen Drüsengewebe in Tätigkeit versetzt werden können.

Wird die eine der zwei Melkpausen verlängert, so ist mehr Raum für die Speicherung der fertigen Milch nötig, vorausgesetzt, dass die Milchleistung des gesamten Tages unverändert bleibt. Dadurch kommt also ein solcher Fall zustande, wo neue Euterabteilungen zur Unterstützung der zuvor arbeitenden herangezogen werden. Und das geschieht anscheinend auch.

Die Theorie, dass je nach Bedarf neue Euterabteilungen zur Tätigkeit mobilisiert werden, erklärt also das bei den oben beschriebenen Versuchen erhaltene Ergebnis, dass die Verlängerung der einen Melkpause und die Verkürzung der anderen keine Veränderung in der je Zeiteinheit entstandenen Milchmenge hervorruft. Ergibt sich doch solchenfalls keine Zunahme des Milchdruckes gegenüber dem vorherigen, obgleich infolge der verlängerten Melkpause bei dem nach ihr vorgenommenen Melken die Milchmenge beträchtlich zunimmt.

Es dürfte begründet sein, nochmals zu bemerken, dass eine derartige ungestörte Milchsekretion trotz veränderten Melkzeiten nur dann möglich ist, wenn diese Veränderung sehr allmählich und regelmässig vor sich geht. Es ist wichtig, dies auch in der Praxis zu beachten, wenn man die Melkzeiten der Herden von den altgewohnten auf andere umzustellen beginnt in der Absicht, die Arbeitsverhältnisse für die Viehpfleger bequemer zu gestalten.

Nach diesen Versuchen zu schliessen, können die Arbeitszeiten mit Rücksicht auf das Melken zum mindesten so angesetzt werden, dass die am reichlichsten erzeugenden Kühe morgens 14 Stunden nach dem Abendmelken und die mittelmässig melkenden 15 Stunden nach dem Abendmelken gemolken werden können. Wenn die am reichlichsten produzierenden Kühe abends zuletzt und morgens zuerst gemolken werden, kann die Nachtruhe der Tierpfleger, was das Melken angeht, etwa 14stündig werden, ohne dass die Leistung der Herde darunter litte.

\section{Schlussfolgerungen}

Der Verfasser hat neulich zwei Versuche über die Wirkung der Dauer der Melkpausen auf die Milchproduktion der Kühe bei zweimaligem Melken ausgeführt 
(6). Die Versuche führten zu solchen Ergebnissen, dass die im Mittel etwa $16 \mathrm{~kg}$ melkenden Versuchskühe dieselbe Milchleistung gaben, unabhängig davon, ob die Pause zwischen Abend- und Morgenmelken 12, 13 oder 15 Stunden, und entsprechend die im Mittel etwa $20 \mathrm{~kg}$ melkenden Versuchskühe auch dieselbe Milchleistung, unabhängig davon, ob die Pause 12, 13 oder 14 Stunden ausgemacht hat. Ein solches Ergebnis ist entweder so zu erklären, dass die Intensität der Milchsekretion ganz unabhängig von der Länge der Melkpause gewesen ist, oder auf Weise, dass diese Intensität während der kürzeren Pause grösser und während der längeren kleiner gewesen ist und dass diese Veränderungen in der Produktionsintensität sich ausgeglichen haben.

Auf Grund des Versuchsmaterials, das in den oben erwähnten Versuchen gesammelt worden ist, hat der Verfasser in dieser Veröffentlichung untersucht, welche der zwei genannten Möglichkeiten in der Wirklichkeit vorlag. Die Berechnungen haben $\mathrm{zu}$ folgenden Ergebnissen geführt:

Das Verlängern der Melkpausen hat, wenn es langsam (nur 5-8 Min. täglich) geschehen ist, die Intensität der Milchsekretion, also die in einer Zeiteinheit gewonnene Milchmenge, gar nicht vermindert, und ihr Verkürzen hat sie nicht vermehrt. Unabhängig von der Dauer der Melkpausen hat man auf die Dauer bei jedesmaligem Melken und bei allen Kühen an Milch eine Menge erhalten, die zu der gesamten Milchleistung des Tages in demselben Verhältnis gestanden hat wie die Melkpause zu 24 Stunden. Die kleinen Abweichungen von dieser Regel, die vorgekommen sind, beruhen offenbar auf Zufall und sind keinesfalls von der Höhe der Leistung abhängig gewesen. Die allgemeine Auffassung, dass das Verlängern der Melkpausen zu einer solchen Steigerung des inneren Milchdruckes im Euter führte, dass er die Milchsekretion verlangsamte, ist also nicht unter den Versuchsverhältnissen zutreffend gewesen. Zur Erklärung der Erscheinung stellt Verfasser die Theorie dar, dass die Kühe im allgemeinen stets über unbenutzte, nicht arbeitende Euterabteilungen verfügen, die bei Bedarf allmählich für die Milchabsonderung mobilisiert werden können, wobei auch die nötige Zunahme des Hohlraumes im Euter zustande kommt. Da dies offenbar nicht in ganz kurzer Zeit geschehen kann, gelangt man zu verschiedenen Ergebnissen, wenn der Übergang von einer Melkpause zur anderen langsam oder schnell herbeigeführt wird. Dies könnte viele scheinbare Widersprüche erklären, die in den Ergebnissen der auf die Lösung dieser Fragen abgesehenen Versuche bestehen.

\section{LITERATUR}

(1) Eskedal, H. Wenzel. 1951. Nogle forsøg med malkning av køer. Beretning fra forsøgslaboratoriet, 255 .

(2) Korkman, Nils. A study of the milk pressure in the teat cisterns of the cow. Annals of the Royal Agric. College of Sweden, 20, p. 303.

(3) Maynard, Leonard, A. 1947. Animal nutrition. 2. edition p. 412.

(4) Petersen, W. E. \& Rigor, T. V. 1932. Relation of pressure to rate and quality of milk secreted. Proc. Soc. Exp. Biol. and Med., 30, p. 254. 
(5) PoIJÄrvi, Ilmari. 1937. Vertailevia kokeita kaksi ja kolme kertaa päivässä lypsämisen vaikutuksesta lehmien maidon- ja voirasvantuotanţoon. (Ref. Vergleichende Versuche über den Einfluss zwei- und dreimal am Tage erfolgenden Melkens auf die Milch- und Butterfetterzeugung der Kühe.) Valt. maat. koet. julk., 94.

(6) - - 1953. Kokeita lypsyjen väliajan vaikutuksesta lehmien maidontuotantoon lypsettäessä kaksi kertaa päivässä. (Ref. Versuche über die Wirkung der Dauer der Melkpausen auf die Milchproduktion der Kühe bei zweimaligem Melken.) Valt. maat. koetoim. tied., 234.

(7) Ragsdale, A. C., Turner, C. W. \& Brody, S. 1924. The rate of milk secretion as affected by an accumulation of milk in the mammary gland. J. Dairy Sci., 7, p. 249.

(8) Swett, W., Fred, W., Graves, R. R., and Creech, G. T., 1932. Quality, size, capacity, gross anatomy, and histology of cow udders in relation to milk production. J. Agr. Research, 45, p. 577 .

(9) Tgelgel, B. 1926. Untersuchungen über den Sekretionsdruck und über das Einschiessen der Milch im Euter des Rindes. Schweizer Arch. f. Tierheilkunde., 68.

SELOSTUS:

LISÄVALAISTUSTA KYSYMYKSEEN LYPSYVÄLIN PITUUDEN VAIKUTUKSESTA LEHMIEN UTAREEN MAIDONERITYKSEN INTENSITEETTIIN

IllMari POIJ ÄRVI

Maatalouskoelaitoksen kotieläinhoito-osasto, Tikkurila

Tekijä on äskettäin julkaissut (6) tuloksia kokeista, joiden lähin tarkoitus oli selvittää lypsyjen erilaisten väliaikojen mahdollista vaikutusta kahdesti päivässä lypsettyjen lehmien maitotuotoksen runsauteen. Osoittautui, että maitotuotos jäi $14-19 \mathrm{~kg}$ lypsävillä lehmillä samaksi, vaikka väli iltalypsystä aamulypsyyn vaihteli $12-15$ tuntiin, ja $14-25 \mathrm{~kg}$ lypsävillä niinikään samaksi, vaikka yöväli vaihteli 12-14 tuntiin. Kun tällainen tulos voisi olla mahdollinen sekä siinä tapauksessa, että sanotut lypsyvälien vaihtelut eivät olisi lainkaan muuttaneet maidon erityksen intensiteettiä, että siinä tapauksessa, että päivävälin lyheneminen olisi lisännyt sitä ja taas yövälin piteneminen sitä vähentänyt, ja että mainitut muutokset olisivat kumonneet toisensa, niin oli kiintoisaa selvittää, kumpi vaihtoehdoista todellisuudessa esiintyi. Tässä tutkimuksessa on edellä mainituissa kokeissa kerääntyneen aineiston perusteella selvitetty juuri lypsyjen väliajan pituuden vaikutusta utareen maidonerityksen intensiteettiin kahdesti päivässä lypsettäessä. Tulokseksi saatiin, että yövälin vaihteleminen niissä rajoissa, kuin edellä mainittiin $(12-15$ t) ei ollenkaan muuttanut maidonerityksen intensiteettiä edellyttäen, että siirtyminen lypsyvälistä toiseen tapahtui hyvin vähitellen, vain $5-8$ min.päivää kohti. Lyspyvälien pituudesta riippumatta on näet 15-20 päivän kuluessa kullakin lypsykerralla ja kaikista lehmistä saatu maitoa määrä, joka on ollut samassa suhteessa koko päivän maitotuotokseen kuin lypsyväli on ollut 24 tuntiin. Ne pienet poikkeamat, mitä tästä säännöstä on ollut, johtuvat ilmeisesti sattumasta, eivätkä ole missään tapauksessa riippuneet lehmien tuotannon runsaudesta. Yleinen käsitys, että lypsyvälin piteneminen johtaisi sellaiseen utareen sisäisen maitopaineen lisääntymiseen, että se hidastaisi maidon eritystä, ei siis koeolosuhteissa ole pitänyt paikkaansa. Selitykseksi ilmiölle tekijä esittää teorian, että lehmillä on yleensä aina käyttämättömiä, toimettomia utarelohkoja, jotka tarpeen sitä vaatiessa voidaan vähitellen mobilisoida maidoneritykseen, jolloin myös tarpeellinen ontelotilavuuden lisäys utareessa saadaan aikaan. Kun tämä ei ilmeisesti voi tapahtua aivan lyhyessä ajassa, johdutaan erilaisiin tuloksiin riippuen siitä, tapahtuuko siirtyminen lypsyvälistä toiseen hitaasti vai nopeasti. Tämä selittäisi monet näennäiset ristiriitaisuudet, joita esiintyy näitä seikkoja selvitelleiden kokeiden tuloksissa. 